Article

\title{
Amino Acid Composition of Milk from Cow, Sheep and Goat Raised in Ailano and Valle Agricola, Two Localities of 'Alto Casertano' (Campania Region)
}

\author{
Nicola Landi, Sara Ragucci (D) and Antimo Di Maro *(D) \\ Department of Environmental, Biological and Pharmaceutical Sciences and Technologies (DiSTABiF), \\ University of Campania 'Luigi Vanvitelli', Via Vivaldi 43, I-81100 Caserta, Italy; \\ nicola.landi@unicampania.it (N.L.); sara.ragucci@unicampania.it (S.R.) \\ * Correspondence: antimo.dimaro@unicampania.it; Tel.: +39-0823-274409
}

Citation: Landi, N.; Ragucci, S.; Di Maro, A. Amino Acid Composition of Milk from Cow, Sheep and Goat Raised in Ailano and Valle Agricola, Two Localities of 'Alto Casertano' (Campania Region). Foods 2021, 10, 2431. https://doi.org/10.3390/ foods10102431

Academic Editor: Francesco Visioli

Received: 10 September 2021

Accepted: 10 October 2021

Published: 13 October 2021

Publisher's Note: MDPI stays neutral with regard to jurisdictional claims in published maps and institutional affiliations.

Copyright: (c) 2021 by the authors. Licensee MDPI, Basel, Switzerland. This article is an open access article distributed under the terms and conditions of the Creative Commons Attribution (CC BY) license (https:// creativecommons.org/licenses/by/ $4.0 /)$.
Abstract: Cow, sheep and goat raw milk raised in Ailano and Valle Agricola territories ('Alto Casertano', Italy) were characterized (raw proteins, free and total amino acids content) to assess milk quality. Raw milk with the highest total protein content is sheep milk followed by goat and cow milk from both localities. Total amino acid content in cow, goat and sheep raw milk is $4.58,4.81$ and $6.62 \mathrm{~g}$ per $100 \mathrm{~g}$, respectively, in which the most abundant amino acid is glutamic acid ( 20.36 g per $100 \mathrm{~g}$ of proteins). Vice versa, the free amino acids content characteristic profiles are different for each species. In particular, the most abundant free amino acid in cow, sheep and goat raw milk is glutamic acid (9.07 $\mathrm{mg}$ per $100 \mathrm{~g}$ ), tyrosine (4.72 $\mathrm{mg}$ per $100 \mathrm{~g}$ ) and glycine (4.54 $\mathrm{mg}$ per $100 \mathrm{~g}$ ), respectively. In addition, goat raw milk is a source of taurine $(14.92 \mathrm{mg}$ per $100 \mathrm{~g})$, retrieved in low amount in cow (1.38 mg per $100 \mathrm{~g}$ ) and sheep ( $2.10 \mathrm{mg}$ per $100 \mathrm{~g}$ ) raw milk. Overall, raw milk from 'Alto Casertano' show a high total protein content and are a good source of essential amino acids.

Keywords: amino acid profile; milk quality; raw proteins; taurine

\section{Introduction}

Milk is a fluid secreted by the female of all mammalian species necessary for the nutritional requirements of the neonate [1]. Milk is an emulsion of oil in water ( $88 \%)$, containing bioactive proteins, lipids and saccharides, as well as main biologically active substances such as antibodies, enzymes, antimicrobial peptides, oligosaccharides and hormones [2]. The main role of milk is to provide energy (lipids and lactose), essential amino acids, fatty acids, vitamins, inorganic elements and water [3,4]. Considering the content of these substances, after childhood humans continue to consume milk from various species such as cattle, goats, sheep, water buffalo, camel, donkey and horse. Moreover, the different technological treatments or transformations of raw milk make this food or its derivatives (e.g., cheese, cream, butter, yogurt and kefir) always available [4].

The protein content of raw milk differs among the species intended for human consumption; indeed, the sheep raw milk has the higher protein content $(5.5 \%)$ followed by water buffalo, camel, cattle, goat, horse and donkey raw milks $(4.4-5.1 \%$; 3.9\%; 3.4\%; $2.9 \% ; 2.5 \%$ and $2.0 \%$, respectively) [5]. In addition, the major contribution in terms of milk nutritional value, consists of caseins $\left(\alpha_{S 1}, \alpha_{S 2}, \beta\right.$ and $\left.\kappa\right)$ and whey protein, rich in essential and non-essential amino acids, having highest biological value, good digestibility, rapid absorption and utilization [6]. For example, in cow, sheep and goat, approximately $80 \%$ of the proteins present in raw milk consists of four proteins named caseins $\left(\alpha_{S 1}, \alpha_{S 2}, \beta\right.$ and $\mathrm{k}$-caseins) [7]. On the other hand, the percentage of each casein changes according to the species. In particular, the total casein content in cow raw milk is $\sim 80 \%$ of total proteins, where $\alpha_{\mathrm{S} 1}, \alpha_{\mathrm{S} 2}, \beta$ and $\kappa$-casein represent $37 \%, 7 \%, 42 \%$ and $9 \%$, respectively [8]. Moreover, the total casein content in sheep raw milk is $85 \%$ of total proteins, where $\alpha_{\mathrm{S} 1}, \alpha_{\mathrm{S} 2}, \beta$ and 
K-casein represent $6.7 \%, 22.8 \%, 61.6 \%$ and $8.9 \%$, respectively, as reported by Balthazar et al., 2017 [9]. The same authors reported that the total casein content in goat raw milk is $65 \%$ of total proteins, where $\alpha_{\mathrm{S} 1}, \beta$ and $\mathrm{k}$-casein represent $5.6 \%, 54.8 \%$ and $20.4 \%$, respectively, while $\alpha_{\mathrm{S} 2}$ (generally $19.2 \%$ ) is highly dependent on the genotype [9].

The remaining $20 \%$ of milk proteins includes major whey proteins $\beta$-lactoglobulin and $\alpha$-lactalbumin as well as other protein constituents: immunoglobulins, serum proteins, milk fat globule proteins, transferrin, lactoferrin, $\beta 2$-microglobulin, several enzymes, peptides and proteolytic products [10]. On the other hand, the protein content of raw milk and their amino acid profiles from various species show great variations both during infant's growth and among species, considering the different growth rate and energy requirements [11]. In addition, genetic, physiological and nutritional factors, as well as environmental conditions, play a great role in these differences $[6,12,13]$.

At the same time, it is known that consumers prefer milk and dairy products, which have favorable sensory qualities, depending on the influence of territorial dietary factors. These differences improve local production of milk and support the conservation of regional resources and related territories [7]. Therefore, acquiring information on the amino acid profile of raw milk proteins from different species is important for an adequate protein uptake and consumption [14] and to add data on the quality of these milk samples.

In this scenario, the present work describes the total protein content (caseins and whey proteins) and the total and free amino acid profiles of raw milk from three different local species found in the mountain community of 'Alto Casertano' (Campania region, Italy). In particular, the study was carried out on cow, sheep and goat lactating breeds raised in the territory of Ailano $\left(41^{\circ} 23^{\prime} \mathrm{N} 14^{\circ} 12^{\prime} \mathrm{E}\right.$; elevation: $\left.260 \mathrm{~m}\right)$ and Valle Agricola $\left(41^{\circ} 25^{\prime} \mathrm{N}\right.$ $14^{\circ} 15^{\prime} \mathrm{E}$; elevation: $\left.691 \mathrm{~m}\right)$, Figure 1.

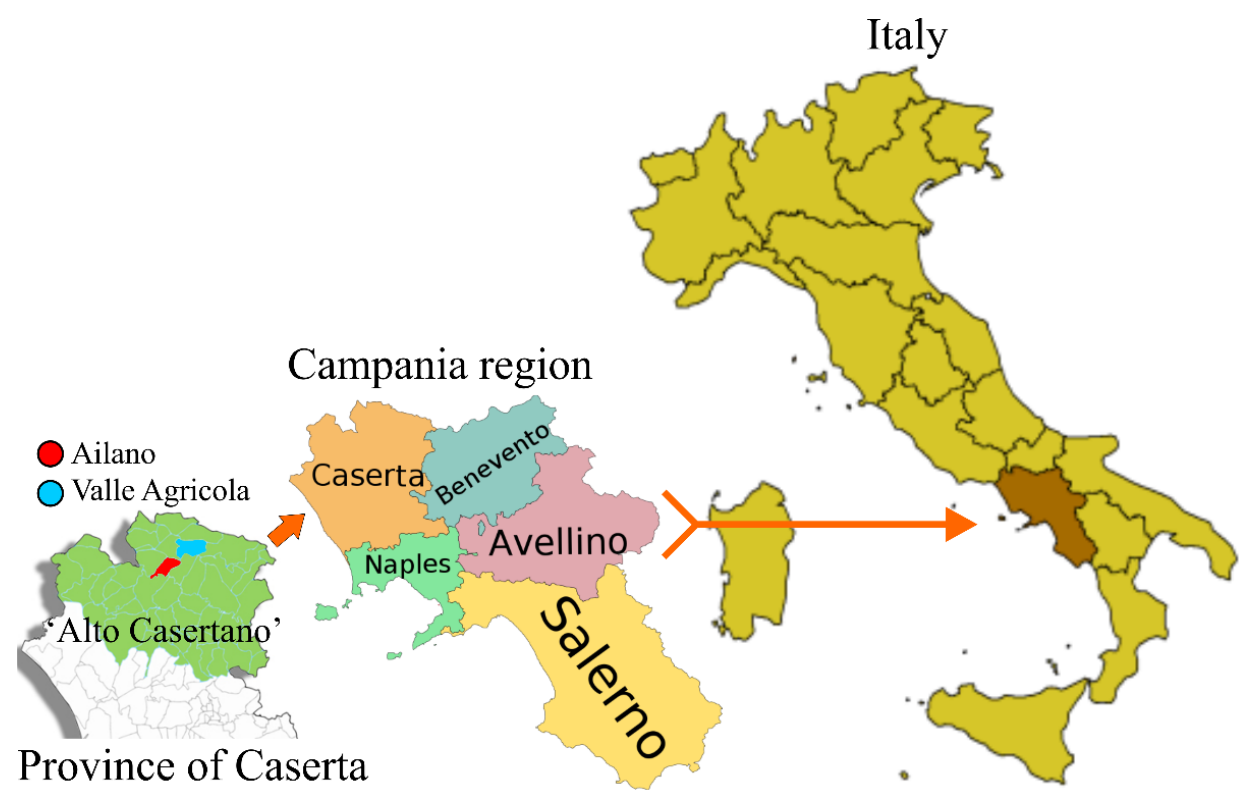

Figure 1. Localities (territory areas) of Ailano and Valle Agricola in which raw milks were sampled are highlighted in red and blue, respectively.

These territories have a rich tradition regarding the breeding of the three species object of this work. Indeed, today there are still small farms located in Ailano and Valle Agricola, where the animals are reared in a semi-wild state. In particular, this type of breeding consists of keeping the animals in the stable during winter and grazing during summer. Furthermore, raw milk produced during summer period is used for the preparation of local dairy products such as 'caciocavallo', 'scamorza', fresh and seasoned 'ricotta', as well as many other kinds of cheese, considered typical products of these territories. Therefore, having more information on the total protein content and the total and free amino acid profile of the raw milk produced in Ailano and Valle Agricola territories ('Alto Casertano') 
could be of interest for the enhancement of raw milk that is also the starting material for the obtainment of typical dairy products.

\section{Materials and Methods}

\subsection{Chemicals and Reagents}

The sources of chemicals used in this work were obtained from Sigma-Aldrich (St. Louis, MO, USA). Chemicals, solvents and reagents for the Kjeldahl method were purchased from Sigma-Aldrich and Carlo Erba Reagents (Milan, Italy). Buffers and reagents for automated amino acid analysis were provided from Biochrom (Cambridge, UK).

\subsection{Milk Samples and Samples Analyzed}

Raw milk samples from cow, sheep and goat (crossbred) were collected from three different farms located in Ailano and Valle Agricola (Caserta, Italy). Each sample was obtained by mixing the raw milk obtained from 20 mares of the crossbred for each species that foaled during the period from February to April. The sampling was made monthly from May to July, repeating the procedure in three random days for each month. Moreover, during the sampling period, the mares were reared on pasture without any form of concentrated integration. The collection procedure followed the norms of good milking practices: the breasts were washed with water and the first three milk jets were discarded in a black bottom mug to verify the presence of lumps. Overall, from each farm, 9 samples of cow, sheep and goat raw milk were collected (27 samples for species from Ailano and Valle Agricola, respectively) and analyzed in triplicate (Scheme 1).

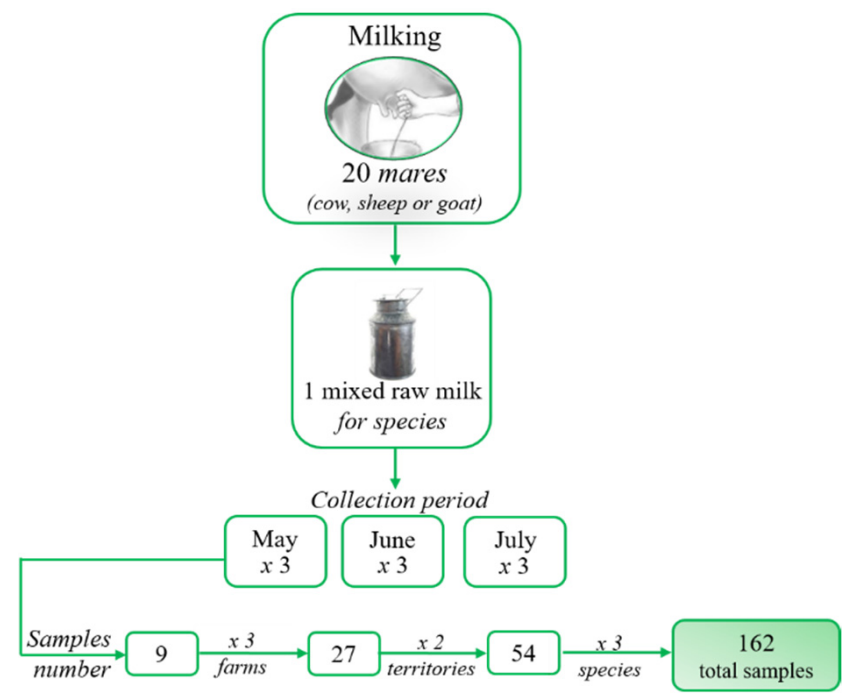

Scheme 1. Schematic representation of raw milk samples collection analyzed.

At the end of milking, homogeneous raw milk samples were collected and stored in polypropylene bottles (Fal-con, Becton Drive, Franklin Lakes, NJ, USA) at $4{ }^{\circ} \mathrm{C}$ during transport. Aliquots of samples were stored at $-80{ }^{\circ} \mathrm{C}$ until use. Therefore, the results reported in all Tables represent the average values for each raw milk.

\subsection{Nitrogen Determination}

Total protein (TP), non-protein nitrogen (NPN) and protein nitrogen (PN) were determined using the Kjeldahl method [15]. For the analysis of TP, about $5.0 \mathrm{~g}$ of raw milk were mineralized using a Mineral Six digester (VWR International PBI, Milan, Italy) and then an Auto Disteam semi-automatic distilling unit (VWR International PBI) was used for determining the nitrogen percentage. Finally, TP was estimated using a nitrogen factor of 6.38.

Subsequently, for the analysis of non-protein nitrogen (NPN; urea, peptides, ammonium, free amino acids and other minor nitrogen containing compounds) and protein 
nitrogen (PN; caseins and whey proteins), about $5.0 \mathrm{~g}$ of raw milk were centrifuged $(3000 \times g)$ in JA.25-50 rotor at $4{ }^{\circ} \mathrm{C}$ for $20 \mathrm{~min}$ in order to remove fat. After filtering on N. 3 Whatman paper, an equal volume of $24 \%$ TCA was added to precipitate proteins. After centrifugation at $3000 \times g$ for $10 \mathrm{~min}$ at $4{ }^{\circ} \mathrm{C}$, protein precipitate was separated from the supernatant filtering on N. 3 Whatman paper. Finally, the precipitated proteins and the supernatant were obtained, containing, respectively, PN and NPN, were analyzed using the Kjeldahl method [15] to estimate the nitrogen percentage.

\subsection{Amino Acid Analysis}

For the analysis of free amino acid composition, three aliquots of $200 \mu \mathrm{L}$ of different raw milk types were precipitated, using $99 \%$ cold ethanol $(800 \mu \mathrm{L})$ in the presence of $200 \mathrm{nmol}$ of nor-Leucine (nor-Leu) as an internal standard, homogenized with a Teflon pestle and centrifuged at $14,000 \times g$ at $4{ }^{\circ} \mathrm{C}$. The supernatants were lyophilized, treated with $3 \%$ sulfosalicylic acid $(500 \mu \mathrm{L})$ to precipitate any protein fraction still present and centrifuged again $[16,17]$. Thus, the supernatants obtained were analyzed.

For the analysis of total amino acid content (free plus protein), $200 \mu \mathrm{L}$ of different raw milk types were freeze-dried and then hydrolyzed with $200 \mu \mathrm{L}$ of $6 \mathrm{~N} \mathrm{HCl}$ containing $0.02 \%$ phenol and nor-Leu $(50 \mathrm{nmol})$ as an internal standard at $110{ }^{\circ} \mathrm{C}$ for $20 \mathrm{~h} \mathrm{[18]}$. Following hydrolysis, $\mathrm{HCl}$ was removed under vacuum and the samples were re-suspended in $0.5 \mathrm{~mL}$ of $0.2 \mathrm{M}$ Li-citrate buffer ( $\mathrm{pH} 2.2$ ).

Aliquots of hydrolyzed and non-hydrolyzed samples were directly analyzed on a Biochrom 30 amino acid analyzer (Biochrom, Cambridge, UK) equipped with a post-column ninhydrin derivatization system [19]. Chemicals and experimental conditions were as suggested by the manufacturer.

\subsection{Cysteine Oxidation with Performic Acid}

Protein milk samples were subjected to oxidation with performic acid. Samples were essentially treated as previously reported [20]. Briefly, $200 \mu \mathrm{L}$ of different raw milk types were freeze-dried and then hydrolyzed in a glass tube and $400 \mu \mathrm{L}$ of performic acid were added. After incubation at $0{ }^{\circ} \mathrm{C}$ for $60 \mathrm{~min}, 200 \mu \mathrm{L}$ of cold $\mathrm{HBr}$ were added. Samples were taken to dryness in a desiccator, rinsed with water and then the hydrolyzed samples were analyzed following the correct procedure.

\subsection{Statistical Analysis}

All the analyses were repeated three times and the data are expressed as mean \pm standard deviation (SD). Data analysis was conducted using Excel Office 2016 (Microsoft Corporation, Redmond, WA, USA). The Bonferroni post-test was used to determine significant differences. The test was performed using a $p<0.05$ using GraphPad Prism 5.0 software (GraphPad Software Inc., San Diego, CA, USA).

\section{Results and Discussion}

\subsection{Total Proteins and Non-Protein Nitrogen in Raw Milk}

Milk is generally considered an important source of proteins present in different content in cow, sheep and goat milk types (3.2, 6.2 and $3.4 \mathrm{~g} / 100 \mathrm{~g}$ of raw milk, respectively). In this study, the average amount of total proteins (TP) as well as protein nitrogen percentage $(\mathrm{PN})$ and non-protein nitrogen percentage (NPN) content of cow, sheep and goat raw milk from 'Ailano' and 'Valle Agricola' territories are shown in Figure 2.

In particular, the TP content (Figure 2a) in Ailano cow raw milk (4.81 g/100 $\mathrm{g}$ of milk) is similar to Valle Agricola cow raw milk (4.60 g/100 g of milk). The TP content of sheep and goat raw milk from 'Ailano' (7.61 and 5.55 g/100 g of milk, respectively) is higher than that of sheep and goat raw milk from 'Valle Agricola' (6.10 and 4.91 g/100 g of milk, respectively). In addition, PN (caseins and whey proteins) was similar in cow raw milk produced from animals raised in both 'Valle Agricola' and 'Ailano' (0.669\% and $0.641 \%$, respectively), while PN was lower in both sheep and goat raw milk produced 
from animals raised in 'Valle Agricola', with respect to 'Ailano' (Figure 2b). Finally, the NPN content of cow and sheep raw milk from 'Ailano' $(0.080 \%)$ is higher than of cow and sheep raw milk from 'Valle Agricola' ( $0.066 \%$ and $0.030 \%$, respectively), while NPN content in goat raw milk is similar for both territories ( $0.049 \%)$ (Figure $2 \mathrm{c})$. The NPN fraction mainly consists of urea, peptides, ammonium, free amino acids and other minor nitrogen containing compounds [21].
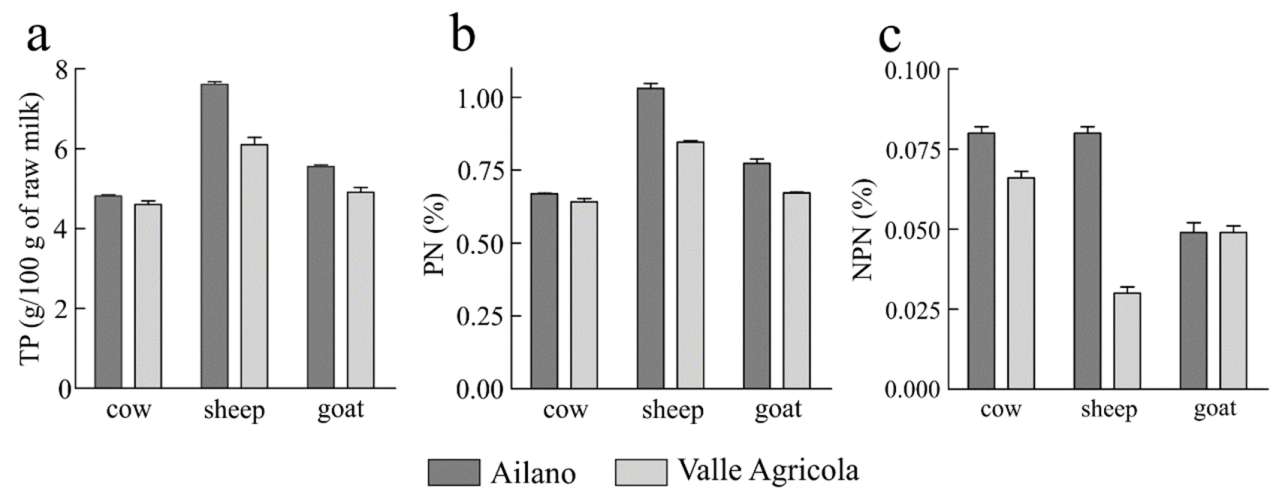

Figure 2. (a) Total protein content (TP), (b) protein nitrogen (PN) and (c) non-protein nitrogen (NPN) content of cow, goat and sheep milk from 'Ailano' and 'Valle Agricola' territories.

\subsection{Amino Acid Content of Cow Raw Milk from 'Ailano' and 'Valle Agricola'}

The total amino acid content (free plus protein) from hydrolyzed cow raw milk obtained by analyzing both 'Ailano' and 'Valle Agricola' samples and their average values are reported in Table 1. Moreover, no statistical differences were retrieved, except for histidine (His) and proline (Pro).

Table 1. Total amino acid composition of cow raw milk from 'Ailano' and 'Valle Agricola'. Values are means $( \pm \mathrm{SD})$ and are expressed as g per $100 \mathrm{~g}$ of milk. See Section 2.2 of Materials and Methods section for more details.

\begin{tabular}{|c|c|c|c|c|c|}
\hline \multicolumn{6}{|c|}{ Total Amino Acid Composition of Cow Raw Milk } \\
\hline Amino Acid & 'Ailano' & 'Valle Agricola' & $p$ Value & Average Values & W.L. Claeys et al. \\
\hline \multicolumn{6}{|c|}{ Essential amino acids } \\
\hline His & $0.24 \pm 0.06 \mathrm{a}$ & $0.11 \pm 0.00 \mathrm{~b}$ & $<0.001$ & 0.17 & 0.10 \\
\hline Ile & $0.19 \pm 0.02 \mathrm{a}$ & $0.18 \pm 0.00 \mathrm{a}$ & $>0.05$ & 0.19 & 0.14 \\
\hline Leu & $0.42 \pm 0.04 \mathrm{a}$ & $0.41 \pm 0.01 \mathrm{a}$ & $>0.05$ & 0.41 & 0.29 \\
\hline Lys & $0.38 \pm 0.01 \mathrm{a}$ & $0.36 \pm 0.01 \mathrm{a}$ & $>0.05$ & 0.37 & 0.27 \\
\hline Met & $0.14 \pm 0.01 \mathrm{a}$ & $0.13 \pm 0.00 \mathrm{a}$ & $>0.05$ & 0.14 & 0.06 \\
\hline Phe & $0.23 \pm 0.02 \mathrm{a}$ & $0.21 \pm 0.00 \mathrm{a}$ & $>0.05$ & 0.22 & 0.16 \\
\hline Thr & $0.24 \pm 0.02 \mathrm{a}$ & $0.22 \pm 0.00 \mathrm{a}$ & $>0.05$ & 0.23 & 0.15 \\
\hline $\operatorname{Trp}$ & n.d. & n.d. & - & - & 0.05 \\
\hline Val & $0.23 \pm 0.02 \mathrm{a}$ & $0.22 \pm 0.00 \mathrm{a}$ & $>0.05$ & 0.23 & 0.16 \\
\hline \multicolumn{6}{|c|}{ Non-essential amino acids } \\
\hline Ala & $0.16 \pm 0.01 \mathrm{a}$ & $0.15 \pm 0.00 \mathrm{a}$ & $>0.05$ & 0.16 & 0.10 \\
\hline Arg & $0.16 \pm 0.02 \mathrm{a}$ & $0.15 \pm 0.00 \mathrm{a}$ & $>0.05$ & 0.16 & 0.11 \\
\hline Asx & $0.35 \pm 0.03 \mathrm{a}$ & $0.35 \pm 0.01 \mathrm{a}$ & $>0.05$ & 0.35 & 0.26 \\
\hline Cys \& & $0.04 \pm 0.00 \mathrm{a}$ & $0.04 \pm 0.00 \mathrm{a}$ & $>0.05$ & 0.04 & 0.02 \\
\hline Glx & $1.02 \pm 0.06 \mathrm{a}$ & $1.01 \pm 0.02 \mathrm{a}$ & $>0.05$ & 1.02 & 0.77 \\
\hline Gly & $0.09 \pm 0.01 \mathrm{a}$ & $0.08 \pm 0.00 \mathrm{a}$ & $>0.05$ & 0.08 & 0.06 \\
\hline Pro & $0.26 \pm 0.03 \mathrm{a}$ & $0.36 \pm 0.00 \mathrm{~b}$ & $<0.001$ & 0.31 & 0.32 \\
\hline Ser & $0.29 \pm 0.03 \mathrm{a}$ & $0.27 \pm 0.01 \mathrm{a}$ & $>0.05$ & 0.28 & 0.16 \\
\hline Tyr & $0.24 \pm 0.02 \mathrm{a}$ & $0.23 \pm 0.00 \mathrm{a}$ & $>0.05$ & 0.23 & 0.15 \\
\hline Total & 4.69 & 4.48 & & 4.58 & 3.33 \\
\hline
\end{tabular}

For protein amino acids, a three-letter code has been used. \& Cys amount was evaluated after performic acid oxidation. n.d., not determined. Values followed by different letters within a row are significantly $(p<0.05)$ different by Bonferroni post-test. 
Subsequently, the average values were compared with those of cow raw milk reported by Claeys et al. [22], showing qualitative and quantitative differences. Considering the 'Alto Casertano' cow milk, Glx (glutamic acid + glutamine; $1.02 \mathrm{~g} / 100 \mathrm{~g}$ ) was the most abundant among total amino acids, followed by leucine $(0.41 \mathrm{~g} / 100 \mathrm{~g})$, lysine $(0.37 \mathrm{~g} / 100 \mathrm{~g})$, Asx (aspartic acid + asparagine; $0.35 \mathrm{~g} / 100 \mathrm{~g})$, proline $(0.31 \mathrm{~g} / 100 \mathrm{~g})$ and serine $(0.28 \mathrm{~g} / 100 \mathrm{~g})$, which represented about $60 \%$ of total amino acids. In addition, the amount of essential amino acids (His, Ile, Leu, Lys, Met, Phe, Thr, Val; -Trp (tryptophan is not included as it was not determined in the total hydrolyzed samples: see Table 1)) in 'Alto Casertano' cow raw milk was $1.96 \mathrm{~g} / 100 \mathrm{~g}$ ( $43 \%$ of total). The amount of methionine and cysteine in 'Alto Casertano' cow raw milk was $0.18 \mathrm{~g} / 100 \mathrm{~g}$ ( $4 \%$ of total), confirming the low level of sulfur amino acids found by Claeys et al. (the amount of sulfur amino acids was $0.10 \mathrm{~g} / 100 \mathrm{~g} ; \sim 3 \%$ of the total). On the other hand, cow milk contains a large amount of glutamic acid, which is $22 \%$ and $23 \%$ for 'Alto Casertano' and milk values reported by Claeys et al., respectively. Furthermore, leucine, lysine, Asx and serine content in 'Alto Casertano' cow raw milk were higher than the milk values reported by Claeys et al., while the proline content is the same $(\sim 0.31 \mathrm{~g} / 100 \mathrm{~g}$ of milk).

In terms of free amino acids, the total amount in 'Alto Casertano' cow raw milk was $21.33 \mathrm{mg} / 100 \mathrm{~g}$ of milk (Table 2) and no statistically significant differences were retrieved, except for glutamic acid (Glu) and urea. Glutamic acid was by far the most abundant among the free protein amino acids $(9.07 \mathrm{mg} / 100 \mathrm{~g}$ of milk). Furthermore, glycine $(1.52 \mathrm{mg} / 100 \mathrm{~g}$ of milk), alanine $(0.91 \mathrm{mg} / 100 \mathrm{~g}$ of milk), aspartic acid $(0.70 \mathrm{mg} / 100 \mathrm{~g}$ of milk), lysine $(0.41 \mathrm{mg} / 100 \mathrm{~g}$ of milk), proline $(0.48 \mathrm{mg} / 100 \mathrm{~g}$ of milk) and arginine $(0.34 \mathrm{mg} / 100 \mathrm{~g}$ of milk) were the most abundant free amino acids in 'Alto Casertano' cow raw milk. On the other hand, the amount of each other protein amino acid did not exceed $1.76 \mathrm{mg} / 100 \mathrm{~g}$ of product ( $8 \%$ of free amino acids total content).

Table 2. Free amino acid composition of cow raw milk from 'Ailano' and 'Valle Agricola'. Values are means $( \pm$ SD) and are expressed as mg per $100 \mathrm{~g}$ of milk. See Section 2.2 of Materials and Methods section for more details.

\begin{tabular}{ccccc}
\hline \multicolumn{5}{c}{ Free Amino Acid Composition of Cow Raw Milk } \\
\hline Amino Acid ${ }^{\#}$ & Ailano & Valle Agricola & $p$ Value & Average Values \\
\hline \multicolumn{5}{c}{ Essential amino acids } \\
His & $0.06 \pm 0.01 \mathrm{a}$ & $0.05 \pm 0.01 \mathrm{a}$ & $>0.05$ & 0.06 \\
Ile & $0.14 \pm 0.01 \mathrm{a}$ & $0.25 \pm 0.01 \mathrm{a}$ & $>0.05$ & 0.20 \\
Leu & $0.14 \pm 0.00 \mathrm{a}$ & $0.23 \pm 0.01 \mathrm{a}$ & $>0.05$ & 0.19 \\
Lys & $0.53 \pm 0.07 \mathrm{a}$ & $0.28 \pm 0.04 \mathrm{a}$ & $>0.05$ & 0.41 \\
Met & n.d. & n.d. & - & - \\
Phe & $0.24 \pm 0.00 \mathrm{a}$ & $0.24 \pm 0.01 \mathrm{a}$ & $>0.05$ & 0.24 \\
Thr & $0.17 \pm 0.08 \mathrm{a}$ & $0.16 \pm 0.00 \mathrm{a}$ & $>0.05$ & 0.17 \\
Trp & n.d. & n.d. & - & - \\
Val & $0.21 \pm 0.00 \mathrm{a}$ & $0.20 \pm 0.01 \mathrm{a}$ & $>0.05$ & 0.21 \\
\hline \multicolumn{5}{c}{ Non-essential amino acids } \\
\hline AAAA & $0.13 \pm 0.00 \mathrm{a}$ & $0.12 \pm 0.01 \mathrm{a}$ & $>0.05$ & 0.13 \\
ABAA & $0.15 \pm 0.02 \mathrm{a}$ & $0.10 \pm 0.02 \mathrm{a}$ & $>0.05$ & 0.13 \\
Ala & $1.01 \pm 0.12 \mathrm{a}$ & $0.80 \pm 0.00 \mathrm{a}$ & $>0.05$ & 0.91 \\
Arg & $0.40 \pm 0.03 \mathrm{a}$ & $0.27 \pm 0.06 \mathrm{a}$ & $>0.05$ & 0.34 \\
Asn & $0.33 \pm 0.02 \mathrm{a}$ & $0.23 \pm 0.02 \mathrm{a}$ & $>0.05$ & 0.28 \\
Asp & $0.68 \pm 0.04 \mathrm{a}$ & $0.71 \pm 0.00 \mathrm{a}$ & $>0.05$ & 0.70 \\
$\beta$-Ala & $0.11 \pm 0.02 \mathrm{a}$ & $0.14 \pm 0.01 \mathrm{a}$ & $>0.05$ & 0.13 \\
Car & n.d & n.d. & - & - \\
Citr & $0.17 \pm 0.01 \mathrm{a}$ & $0.11 \pm 0.01 \mathrm{a}$ & $>0.05$ & 0.14 \\
Cys & n.d. & n.d & - & - \\
\hline
\end{tabular}


Table 2. Cont.

\begin{tabular}{ccccc}
\hline \multicolumn{5}{c}{ Free Amino Acid Composition of Cow Raw Milk } \\
\hline Amino Acid $^{\#}$ & Ailano & Valle Agricola & $p$ Value & Average Values \\
\hline Ethan & $1.49 \pm 0.30 \mathrm{a}$ & $0.82 \pm 0.14 \mathrm{a}$ & $>0.05$ & 1.16 \\
GABA & n.d. & $0.02 \pm 0.00$ & $>0.05$ & - \\
Gln & n.d. & n.d. & - & - \\
Glu & $6.18 \pm 0.21 \mathrm{a}$ & $11.96 \pm 0.18 \mathrm{~b}$ & $<0.001$ & 9.07 \\
Gly & $1.39 \pm 0.05 \mathrm{a}$ & $1.65 \pm 0.03 \mathrm{a}$ & $>0.05$ & 1.52 \\
Homocys & n.d. & n.d. & - & - \\
1-Mhis & n.d & n.d. & - & - \\
3-Mhis & n.d. & n.d. & - & 0.08 \\
Orn & $0.06 \pm 0.00 \mathrm{a}$ & $0.09 \pm 0.00 \mathrm{a}$ & $>0.05$ & 1.57 \\
Pea & $1.19 \pm 0.07 \mathrm{a}$ & $1.94 \pm 0.08 \mathrm{a}$ & $>0.05$ & 1.35 \\
Phser & $1.34 \pm 0.03 \mathrm{a}$ & $1.36 \pm 0.01 \mathrm{a}$ & $>0.05$ & 0.48 \\
Pro & $0.58 \pm 0.08 \mathrm{a}$ & $0.37 \pm 0.03 \mathrm{a}$ & $>0.05$ & 0.14 \\
Sarc & $0.13 \pm 0.00 \mathrm{a}$ & $0.15 \pm 0.01 \mathrm{a}$ & $>0.05$ & 0.30 \\
Ser & $0.30 \pm 0.02 \mathrm{a}$ & $0.30 \pm 0.04 \mathrm{a}$ & $>0.05$ & 1.38 \\
Taur & $1.39 \pm 0.02 \mathrm{a}$ & $1.37 \pm 0.05 \mathrm{a}$ & $>0.05$ & 0.11 \\
Tyr & $0.18 \pm 0.06 \mathrm{a}$ & $0.03 \pm 0.01 \mathrm{a}$ & $>0.05$ & $\mathbf{2 1 . 4 0}$ \\
\hline Total & $\mathbf{1 8 . 7 0}$ & $\mathbf{2 3 . 9 5}$ & - & 43.87 \\
\hline Urea & $46.58 \pm 1.00 \mathrm{a}$ & $41.16 \pm 3.24 \mathrm{~b}$ & $<0.001$ & \\
\hline
\end{tabular}

\# Free and protein amino acids. n.d., not detected. Three-letter code has been used. Values followed by different letters within a row are significantly $(p<0.05)$ different by Bonferroni post-test. Protein amino acids are highlighted in bold.

The analysis also evidenced the presence of ten non-protein amino acids (i.e., $L-\alpha$ aminoadipic acid (AAAA); L- $\alpha$-aminobutyric acid (ABAA); $\beta$-alanine ( $\beta$-Ala); L-citrulline (Citr); ethanolamine (Ethan); L-ornithine (Orn); phosphorylethanolamine (Pea); phosphoserine (Phser); L-sarcosine (Sarc) and taurine (Taur)). The amount of these non-protein amino acids was $6.21 \mathrm{mg} / 100 \mathrm{~g}$ of milk ( $29 \%$ of total). Finally, the analysis reveals that urea content in 'Alto Casertano' raw milk ( $43.87 \mathrm{mg} / 100 \mathrm{~g}$ of milk) represents about $10 \%$ of the non-protein nitrogen (NPN) fraction from 'Alto Casertano' cow raw milk, while free amino acids represent about $5 \%$ of NPN fraction.

\subsection{Amino Acid Content of Sheep Raw Milk from 'Ailano' and 'Valle Agricola'}

Total amino acid content (free plus protein) from hydrolyzed sheep raw milk obtained by analyzing both 'Ailano' and 'Valle Agricola' samples and their average values were reported in Table 3. Moreover, no statistical differences were retrieved, except for some amino acids (i.d.: Ile, Leu, Lys, Phe, Thr, Val, Asx, Glx, Pro, Ser and Tyr). Subsequently, the average values were compared with those of sheep milk reported by Claeys et al. [22], showing qualitative and quantitative differences. In particular, Glx (glutamic acid + glutamine; $1.35 \mathrm{~g} / 100 \mathrm{~g}$ ) was the most abundant among the total amino acids in 'Alto Casertano' sheep raw milk, followed by proline $(0.64 \mathrm{~g} / 100 \mathrm{~g})$, leucine $(0.54 \mathrm{~g} / 100 \mathrm{~g})$, lysine $(0.51 \mathrm{~g} / 100 \mathrm{~g})$, Asx (aspartic acid + asparagine; $0.50 \mathrm{~g} / 100 \mathrm{~g}$ ) and serine $(0.39 \mathrm{~g} / 100 \mathrm{~g}$ ), which represent about $59 \%$ of the total amino acids.

In addition, the amount of essential amino acids (His, Ile, Leu, Lys, Met, Phe, Thr, Val; -Trp (tryptophan is not included as it was not determined in the total hydrolyzed samples: see Table 3)) in 'Alto Casertano' sheep raw milk was $2.72 \mathrm{~g} / 100 \mathrm{~g}$ ( $41 \%$ of total). The amount of methionine and cysteine in 'Alto Casertano' sheep raw milk was $0.26 \mathrm{~g} / 100 \mathrm{~g}$ ( $4.0 \%$ of total), confirming the low level of sulfur amino acids found by Claeys et al. (the amount of sulfur amino acids was $0.20 \mathrm{~g} / 100 \mathrm{~g} ; \sim 3 \%$ of the total). 
Table 3. Total amino acid composition of sheep raw milk from 'Ailano' and 'Valle Agricola'. Values are means ( \pm SD) and are expressed as g per $100 \mathrm{~g}$ of milk. See Section 2.2 of Materials and Methods section for more details.

\begin{tabular}{|c|c|c|c|c|c|}
\hline \multicolumn{6}{|c|}{ Total Amino Acid Composition of Sheep Raw Milk } \\
\hline Amino Acid & 'Ailano' & 'Valle Agricola' & $p$ Value & Average Values & W.L. Claeys et al. \\
\hline \multicolumn{6}{|c|}{ Essential amino acids } \\
\hline His & $0.19 \pm 0.00 \mathrm{a}$ & $0.15 \pm 0.01 \mathrm{a}$ & $>0.05$ & 0.17 & 0.17 \\
\hline Ile & $0.32 \pm 0.00 \mathrm{a}$ & $0.25 \pm 0.01 \mathrm{~b}$ & $<0.01$ & 0.29 & 0.34 \\
\hline Leu & $0.58 \pm 0.00 \mathrm{a}$ & $0.50 \pm 0.02 b$ & $<0.01$ & 0.54 & 0.59 \\
\hline Lys & $0.54 \pm 0.00 \mathrm{a}$ & $0.48 \pm 0.06 \mathrm{~b}$ & $<0.05$ & 0.51 & 0.51 \\
\hline Met & $0.21 \pm 0.00 \mathrm{a}$ & $0.18 \pm 0.01 \mathrm{a}$ & $>0.05$ & 0.19 & 0.16 \\
\hline Phe & $0.37 \pm 0.00 \mathrm{a}$ & $0.28 \pm 0.01 \mathrm{~b}$ & $<0.001$ & 0.33 & 0.28 \\
\hline Thr & $0.38 \pm 0.01 \mathrm{a}$ & $0.31 \pm 0.01 b$ & $<0.01$ & 0.35 & 0.27 \\
\hline $\operatorname{Trp}$ & n.d. & n.d. & - & - & 0.08 \\
\hline Val & $0.39 \pm 0.00 \mathrm{a}$ & $0.30 \pm 0.01 \mathrm{~b}$ & $<0.001$ & 0.34 & 0.45 \\
\hline \multicolumn{6}{|c|}{ Non-essential amino acids } \\
\hline Ala & $0.27 \pm 0.00 \mathrm{a}$ & $0.23 \pm 0.02 \mathrm{a}$ & $>0.05$ & 0.25 & 0.27 \\
\hline Arg & $0.26 \pm 0.00 \mathrm{a}$ & $0.21 \pm 0.01 \mathrm{a}$ & $>0.05$ & 0.23 & 0.20 \\
\hline Asx & $0.55 \pm 0.00 \mathrm{a}$ & $0.45 \pm 0.03 b$ & $<0.001$ & 0.50 & 0.33 \\
\hline Cys \& & $0.09 \pm 0.01 \mathrm{a}$ & $0.05 \pm 0.01 \mathrm{a}$ & $>0.05$ & 0.07 & 0.04 \\
\hline Glx & $1.45 \pm 0.00 \mathrm{a}$ & $1.25 \pm 0.10 \mathrm{~b}$ & $<0.001$ & 1.35 & 1.02 \\
\hline Gly & $0.13 \pm 0.00 \mathrm{a}$ & $0.11 \pm 0.01 \mathrm{a}$ & $>0.05$ & 0.12 & 0.04 \\
\hline Pro & $0.74 \pm 0.00 \mathrm{a}$ & $0.53 \pm 0.03 b$ & $<0.001$ & 0.64 & 0.58 \\
\hline Ser & $0.43 \pm 0.01 \mathrm{a}$ & $0.35 \pm 0.02 b$ & $<0.01$ & 0.39 & 0.49 \\
\hline Tyr & $0.38 \pm 0.00 \mathrm{a}$ & $0.31 \pm 0.01 b$ & $<0.01$ & 0.34 & 0.28 \\
\hline Total & 7.30 & 5.93 & - & 6.62 & 6.09 \\
\hline
\end{tabular}

For protein amino acids, a three-letter code has been used. \& Cys amount was evaluated after performic acid oxidation. n.d., not detected.

Values followed by different letters within a row are significantly $(p<0.05)$ different by Bonferroni post-test.

On the other hand, sheep raw milk contains a large amount of glutamic acid, which is $20 \%$ and $17 \%$ for 'Alto Casertano' and milk values reported by Claeys et al., respectively. Furthermore, proline, Asx, threonine and phenylalanine content in 'Alto Casertano' sheep raw milk were higher than in milk reported by Claeys et al., while the other amino acids were present in lower quantities in 'Alto Casertano' sheep raw milk, compared to the information reported by Claeys et al. [22].

In terms of free amino acids, the total amount in 'Alto Casertano' sheep raw milk was $21.86 \mathrm{mg} / 100 \mathrm{~g}$ of milk (Table 4) and no statistically significant differences were found, except for glutamic acid (Glu), taurine (Taur), tyrosine (Tyr) and urea. Tyrosine was by far the most abundant among free protein amino acids $(4.72 \mathrm{mg} / 100 \mathrm{~g}$ of milk), followed by Glu (glutamic acid; $2.98 \mathrm{mg} / 100 \mathrm{~g}$ of milk), glycine (1.12 mg/100 $\mathrm{g}$ of milk), asparagine $(0.77 \mathrm{mg} / 100 \mathrm{~g}$ of milk) phenylalanine $(0.73 \mathrm{mg} / 100 \mathrm{~g}$ of milk), arginine $(0.71 \mathrm{mg} / 100 \mathrm{~g}$ of milk) and alanine $(0.60 \mathrm{mg} / 100 \mathrm{~g}$ of milk).

On the other hand, the amount of each other protein amino acid did not exceed $2.8 \mathrm{mg} / 100 \mathrm{~g}$ of product ( $13 \%$ of free amino acids total content). The analysis also revealed the presence of twelve non-protein amino acids (i.e., $\mathrm{L}-\alpha$-aminoadipic acid (AAAA); $\beta$-alanine ( $\beta$-Ala); L-carnitine (Car); L-citrulline (Citr); ethanolamine (Ethan); 1-Methylhistidine (1-Mhis); 3-Methylhistidine (3-Mis); L-ornithine (Orn); phosphorylethanolamine (Pea); phosphoserine (Phser); L-sarcosine (Sarc); taurine (Taur)). The amount of these non-protein amino acids was $7.47 \mathrm{mg} / 100 \mathrm{~g}$ of milk ( $34 \%$ of total). Finally, the analysis reveals that urea content in 'Alto Casertano' raw milk (59.60 mg/100 g of milk) represents about $17 \%$ of the NPN fraction from 'Alto Casertano' sheep raw milk, while free amino acids represent about $6 \%$ of the NPN fraction. 
Table 4. Free amino acid composition of sheep raw milk from 'Ailano' and 'Valle Agricola'. Values are means $( \pm \mathrm{SD})$ and are expressed as mg per $100 \mathrm{~g}$ of milk. See Section $2.2 \mathrm{of} \mathrm{Materials} \mathrm{and} \mathrm{Methods}$ section for more details.

\begin{tabular}{|c|c|c|c|c|}
\hline \multicolumn{5}{|c|}{ Free Amino Acid Composition of Sheep Raw Milk } \\
\hline Amino Acid ${ }^{\#}$ & Ailano & Valle Agricola & $p$ Value & Average Values \\
\hline \multicolumn{5}{|c|}{ Essential amino acids } \\
\hline His & $0.09 \pm 0.00 \mathrm{a}$ & $0.13 \pm 0.01 \mathrm{a}$ & $>0.05$ & 0.11 \\
\hline Ile & $0.21 \pm 0.01 \mathrm{a}$ & $0.23 \pm 0.03 \mathrm{a}$ & $>0.05$ & 0.22 \\
\hline Leu & $0.56 \pm 0.05 \mathrm{a}$ & $0.30 \pm 0.03 \mathrm{a}$ & $>0.05$ & 0.43 \\
\hline Lys & $0.29 \pm 0.00 \mathrm{a}$ & $0.24 \pm 0.01 \mathrm{a}$ & $>0.05$ & 0.27 \\
\hline Met & $0.03 \pm 0.00$ & n.d. & $>0.05$ & - \\
\hline Phe & $1.11 \pm 0.03 \mathrm{a}$ & $0.35 \pm 0.01 \mathrm{a}$ & $>0.05$ & 0.73 \\
\hline Thr & $0.64 \pm 0.02 \mathrm{a}$ & $0.28 \pm 0.00 \mathrm{a}$ & $>0.05$ & 0.46 \\
\hline $\operatorname{Trp}$ & n.d. & n.d. & - & 0.00 \\
\hline Val & $0.47 \pm 0.00 \mathrm{a}$ & $0.36 \pm 0.00 \mathrm{a}$ & $>0.05$ & 0.42 \\
\hline \multicolumn{5}{|c|}{ Non-essential amino acids } \\
\hline AAAA & $0.14 \pm 0.00 \mathrm{a}$ & $0.10 \pm 0.02 \mathrm{a}$ & $>0.05$ & 0.12 \\
\hline ABAA & $0.06 \pm 0.01$ & n.d. & $>0.05$ & - \\
\hline Ala & $0.81 \pm 0.00 \mathrm{a}$ & $0.39 \pm 0.00 \mathrm{a}$ & $>0.05$ & 0.60 \\
\hline Arg & $0.78 \pm 0.03 \mathrm{a}$ & $0.63 \pm 0.01 \mathrm{a}$ & $>0.05$ & 0.71 \\
\hline Asn & $0.60 \pm 0.13 \mathrm{a}$ & $0.93 \pm 0.04 \mathrm{a}$ & $>0.05$ & 0.77 \\
\hline Asp & $0.39 \pm 0.01 \mathrm{a}$ & $0.27 \pm 0.01 \mathrm{a}$ & $>0.05$ & 0.33 \\
\hline$\beta$-Ala & $0.25 \pm 0.03 \mathrm{a}$ & $0.13 \pm 0.02 \mathrm{a}$ & $>0.05$ & 0.19 \\
\hline Car & $0.08 \pm 0.01 \mathrm{a}$ & $0.09 \pm 0.00 \mathrm{a}$ & $>0.05$ & 0.09 \\
\hline Citr & $0.55 \pm 0.01 \mathrm{a}$ & $0.39 \pm 0.05 a$ & $>0.05$ & 0.47 \\
\hline Cys & n.d. & n.d. & - & - \\
\hline Ethan & $2.03 \pm 0.00 \mathrm{a}$ & $1.62 \pm 0.12 \mathrm{a}$ & $>0.05$ & 1.83 \\
\hline GABA & $0.02 \pm 0.01$ & n.d. & $>0.05$ & 0.01 \\
\hline Gln & n.d. & n.d. & - & - \\
\hline Glu & $4.08 \pm 0.07 \mathrm{a}$ & $1.87 \pm 0.04 b$ & $<0.001$ & 2.98 \\
\hline Gly & $1.35 \pm 0.02 \mathrm{a}$ & $0.89 \pm 0.08 \mathrm{a}$ & $>0.05$ & 1.12 \\
\hline Homocys & $0.03 \pm 0.01$ & n.d. & $>0.05$ & - \\
\hline 1-Mhis & $0.21 \pm 0.00 \mathrm{a}$ & $0.40 \pm 0.03 \mathrm{a}$ & $>0.05$ & 0.31 \\
\hline 3-Mhis & $0.09 \pm 0.04 \mathrm{a}$ & $0.19 \pm 0.02 \mathrm{a}$ & $>0.05$ & 0.14 \\
\hline Orn & $0.15 \pm 0.00 \mathrm{a}$ & $0.14 \pm 0.00 \mathrm{a}$ & $>0.05$ & 0.15 \\
\hline Pea & $0.50 \pm 0.01 \mathrm{a}$ & $0.08 \pm 0.02 \mathrm{a}$ & $>0.05$ & 0.29 \\
\hline Phser & $1.90 \pm 0.03 \mathrm{a}$ & $1.35 \pm 0.07 a$ & $>0.05$ & 1.63 \\
\hline Pro & $0.43 \pm 0.02 \mathrm{a}$ & $0.13 \pm 0.02 \mathrm{a}$ & $>0.05$ & 0.28 \\
\hline Sarc & $0.15 \pm 0.03 a$ & $0.14 \pm 0.00 \mathrm{a}$ & $>0.05$ & 0.15 \\
\hline Ser & $0.31 \pm 0.06 \mathrm{a}$ & $0.25 \pm 0.02 \mathrm{a}$ & $>0.05$ & 0.28 \\
\hline Taur & $0.42 \pm 0.02 \mathrm{a}$ & $3.78 \pm 0.11 b$ & $<0.001$ & 2.10 \\
\hline Tyr & $9.12 \pm 0.09 \mathrm{a}$ & $0.32 \pm 0.05 b$ & $<0.001$ & 4.72 \\
\hline Total & 27.85 & 15.98 & - & 21.86 \\
\hline Urea & $81.30 \pm 1.80 \mathrm{a}$ & $37.90 \pm 1.61 \mathrm{~b}$ & $<0.001$ & 59.6 \\
\hline
\end{tabular}

\# Free and protein amino acids. n.d., not detected. Three-letter code has been used. Values followed by different letters within a row are significantly $(p<0.05)$ different by Bonferroni post-test. Protein amino acids are highlighted in bold.

\subsection{Amino Acid Content of Goat Raw Milk from 'Ailano' and 'Valle Agricola'}

The total amino acid content (free plus protein) of hydrolyzed goat raw milk obtained by analyzing both 'Ailano' and 'Valle Agricola' samples and their average values are reported in Table 5; moreover, no statistically significant differences were found, except for Glx (glutamic acid + glutamine). Subsequently, the average values were compared with those of the goat milk reported by Claeys et al. [22], showing qualitative and quantitative differences. In particular, Glx (glutamic acid + glutamine; $1.03 \mathrm{~g} / 100 \mathrm{~g}$ ) was the most abundant among the total amino acids in 'Alto Casertano' goat row milk, followed by proline $(0.47 \mathrm{~g} / 100 \mathrm{~g})$, leucine $(0.43 \mathrm{~g} / 100 \mathrm{~g})$, lysine $(0.37 \mathrm{~g} / 100 \mathrm{~g})$ and Asx (aspartic 
acid + asparagine; $0.34 \mathrm{~g} / 100 \mathrm{~g}$ ), which represent about $55 \%$ of total amino acids. In addition, the amount of essential amino acids (His, Ile, Leu, Lys, Met, Phe, Thr, Val, -Trp (tryptophan is not included as it was not determined in the total hydrolysed samples: see Table 5)) in 'Alto Casertano' goat raw milk was $2.08 \mathrm{~g} / 100 \mathrm{~g}$ ( $\sim 3 \%$ of total). The amount of methionine and cysteine in 'Alto Casertano' goat raw milk was $0.19 \mathrm{~g} / 100 \mathrm{~g}(\sim 4.0 \%$ of total), confirming the low level of sulfur amino acids found by Claeys et al. [22] (the amount of sulfur amino acids was $0.13 \mathrm{~g} / 100 \mathrm{~g} ; 4 \%$ of the total).

Table 5. Total amino acid composition of goat raw milk from 'Ailano' and 'Valle Agricola'. Values are means ( \pm SD) and are expressed as g per $100 \mathrm{~g}$ of milk. See Section $2.2 \mathrm{of} \mathrm{Materials} \mathrm{and} \mathrm{Methods} \mathrm{section} \mathrm{for} \mathrm{more} \mathrm{details.}$

\begin{tabular}{|c|c|c|c|c|c|}
\hline \multicolumn{6}{|c|}{ Total Amino Acid Composition of Goat Raw Milk } \\
\hline Amino Acid & 'Ailano' & 'Valle Agricola' & $p$ Value & Average Values & W.L. Claeys et al. \\
\hline \multicolumn{6}{|c|}{ Essential amino acids } \\
\hline His & $0.13 \pm 0.02 \mathrm{a}$ & $0.11 \pm 0.00 \mathrm{a}$ & $>0.05$ & 0.12 & 0.10 \\
\hline Ile & $0.22 \pm 0.03 \mathrm{a}$ & $0.19 \pm 0.01 \mathrm{a}$ & $>0.05$ & 0.21 & 0.21 \\
\hline Leu & $0.46 \pm 0.03 \mathrm{a}$ & $0.40 \pm 0.02 \mathrm{a}$ & $>0.05$ & 0.43 & 0.31 \\
\hline Lys & $0.41 \pm 0.03 \mathrm{a}$ & $0.34 \pm 0.01 \mathrm{~b}$ & $<0.05$ & 0.37 & 0.29 \\
\hline Met & $0.14 \pm 0.01 \mathrm{a}$ & $0.14 \pm 0.01 \mathrm{a}$ & $>0.05$ & 0.14 & 0.08 \\
\hline Phe & $0.26 \pm 0.03 a$ & $0.23 \pm 0.01 \mathrm{a}$ & $>0.05$ & 0.25 & 0.16 \\
\hline Thr & $0.30 \pm 0.03 a$ & $0.28 \pm 0.01 \mathrm{a}$ & $>0.05$ & 0.29 & 0.24 \\
\hline $\operatorname{Trp}$ & n.d. & n.d. & - & - & 0.04 \\
\hline Val & $0.28 \pm 0.03 \mathrm{a}$ & $0.26 \pm 0.01 \mathrm{a}$ & $>0.05$ & 0.27 & 0.24 \\
\hline \multicolumn{6}{|c|}{ Non-essential amino acids } \\
\hline Ala & $0.17 \pm 0.03$ & $0.13 \pm 0.01$ & $>0.05$ & 0.15 & 0.12 \\
\hline Arg & $0.16 \pm 0.02$ & $0.13 \pm 0.01$ & $>0.05$ & 0.14 & 0.12 \\
\hline Asx & $0.39 \pm 0.03$ & $0.30 \pm 0.01$ & $<0.01$ & 0.34 & 0.21 \\
\hline Cys \& & $0.05 \pm 0.01$ & $0.04 \pm 0.00$ & $>0.05$ & 0.05 & 0.05 \\
\hline Glx & $1.08 \pm 0.09$ & $0.98 \pm 0.03$ & $<0.001$ & 1.03 & 0.63 \\
\hline Gly & $0.09 \pm 0.01$ & $0.07 \pm 0.00$ & $>0.05$ & 0.08 & 0.05 \\
\hline Pro & $0.49 \pm 0.05$ & $0.45 \pm 0.03$ & $>0.05$ & 0.47 & 0.37 \\
\hline Ser & $0.27 \pm 0.04$ & $0.27 \pm 0.01$ & $>0.05$ & 0.27 & 0.18 \\
\hline Tyr & $0.22 \pm 0.02$ & $0.17 \pm 0.01$ & $>0.05$ & 0.20 & 0.18 \\
\hline Total & 5.13 & 4.48 & - & 4.81 & 3.57 \\
\hline
\end{tabular}

For protein amino acids, a three-letter code has been used. \& Cys amount was evaluated after performic acid oxidation. n.d. not determined. Values followed by different letters within a row are significantly $(p<0.05)$ different by Bonferroni post-test.

On the other hand, goat raw milk contains a large amount of glutamic acid, which is $21 \%$ and $18 \%$ for 'Alto Casertano' and the milk values reported by Claeys et al., respectively. Furthermore, the proline, Asx, threonine, leucine lysine and phenylalanine content in 'Alto Casertano' goat raw milk were higher than the milk values reported by Claeys et al., while the other amino acids were present in lower quantities in 'Alto Casertano' goat raw milk compared to in the milk values reported by Claeys et al. [22].

In terms of free amino acids, the total amount in 'Alto Casertano' goat raw milk was $46.15 \mathrm{mg} / 100 \mathrm{~g}$ of milk (Table 6), and no statistically significant differences were found except for taurine (Taur) and urea. Glycine was by far the most abundant among free protein amino acids ( $4.54 \mathrm{mg} / 100 \mathrm{~g}$ of milk; about $10 \%$ of total), followed by glutamic acid $(4.12 \mathrm{mg} / 100 \mathrm{~g})$, asparagine (2.75 mg/100 g of milk), glutamine (2.15 mg/100 $\mathrm{g}$ of milk), serine $(1.63 \mathrm{mg} / 100 \mathrm{~g})$ and alanine $(1.26 \mathrm{mg} / 100 \mathrm{~g})$.

On the other hand, the amount of each other protein amino acid did not exceed $5.8 \mathrm{mg} / 100 \mathrm{~g}$ of the product $(\sim 12 \%$ of free amino acids total content). The analysis also revealed the presence of twelve non-protein amino acids (i.e., L- $\alpha$-aminoadipic acid (AAAA); L- $\alpha$-amminobutirrico (ABAA); $\beta$-alanine ( $\beta$-Ala); L-citrulline (Citr); ethanolamine (Ethan); 1-Methylhistidine (1-Mhis); 3-Methylhistidine (3-Mis); L-ornithine (Orn); phosphorylethanolamine (Pea); phosphoserine (Phser); L-sarcosine (Sarc); taurine (Taur)). The amount of these non-protein amino acids was $24.0 \mathrm{mg} / 100 \mathrm{~g}$ of milk ( $52 \%$ of total). Finally, 
the analysis revealed that urea content in 'Alto Casertano' raw milk $(67.88 \mathrm{mg} / 100 \mathrm{~g}$ of milk) represents about $22 \%$ of the NPN fraction from 'Alto Casertano' goat raw milk, while free amino acids represent about $15 \%$ of the NPN fraction.

Table 6. Free amino acid composition of goat raw milk from 'Ailano' and 'Valle Agricola'. Values are means $( \pm \mathrm{SD})$ and are expressed as mg per $100 \mathrm{~g}$ of milk. See Section $2.2 \mathrm{of}$ Materials and Methods section for more details.

\begin{tabular}{|c|c|c|c|c|}
\hline \multicolumn{5}{|c|}{ Free Amino Acid Composition of Goat Raw Milk } \\
\hline Amino acid ${ }^{\#}$ & Ailano & Valle Agricola & $p$ Value & Average Values \\
\hline \multicolumn{5}{|c|}{ Essential amino acids } \\
\hline His & $0.05 \pm 0.00 \mathrm{a}$ & $0.16 \pm 0.01 \mathrm{a}$ & $>0.05$ & 0.11 \\
\hline Ile & $0.19 \pm 0.01 \mathrm{a}$ & $0.37 \pm 0.04 \mathrm{a}$ & $>0.05$ & 0.28 \\
\hline Leu & $0.25 \pm 0.10 \mathrm{a}$ & $0.67 \pm 0.07 \mathrm{a}$ & $>0.05$ & 0.46 \\
\hline Lys & $0.51 \pm 0.02 \mathrm{a}$ & $0.78 \pm 0.00 \mathrm{a}$ & $>0.05$ & 0.65 \\
\hline Met & n.d. & n.d. & - & - \\
\hline Phe & $0.39 \pm 0.01 \mathrm{a}$ & $0.49 \pm 0.01 \mathrm{a}$ & $>0.05$ & 0.44 \\
\hline Thr & $0.84 \pm 0.01 \mathrm{a}$ & $0.54 \pm 0.03 \mathrm{a}$ & $>0.05$ & 0.69 \\
\hline Trp & n.d. & n.d & - & - \\
\hline Val & $0.63 \pm 0.01 \mathrm{a}$ & $0.98 \pm 0.01 \mathrm{a}$ & $>0.05$ & 0.81 \\
\hline \multicolumn{5}{|c|}{ Non-essential amino acids } \\
\hline AAAA & $0.08 \pm 0.01 \mathrm{a}$ & $0.14 \pm 0.05 \mathrm{a}$ & $>0.05$ & 0.11 \\
\hline ABAA & $0.31 \pm 0.00 \mathrm{a}$ & $0.10 \pm 0.01 \mathrm{a}$ & $>0.05$ & 0.21 \\
\hline Ala & $1.33 \pm 0.04 \mathrm{a}$ & $1.19 \pm 0.02 \mathrm{a}$ & $>0.05$ & 1.26 \\
\hline Arg & $0.48 \pm 0.02 \mathrm{a}$ & $1.24 \pm 0.03 \mathrm{a}$ & $>0.05$ & 0.86 \\
\hline Asn & $1.89 \pm 0.43 \mathrm{a}$ & $3.60 \pm 0.02 \mathrm{a}$ & $>0.05$ & 2.75 \\
\hline Asp & $0.87 \pm 0.00 \mathrm{a}$ & $0.33 \pm 0.02 \mathrm{a}$ & $>0.05$ & 0.60 \\
\hline$\beta$-Ala & $0.18 \pm 0.00 \mathrm{a}$ & $0.07 \pm 0.00 \mathrm{a}$ & $>0.05$ & 0.13 \\
\hline Car & n.d. & n.d. & - & - \\
\hline Citr & $1.67 \pm 0.04 \mathrm{a}$ & $1.39 \pm 0.04 \mathrm{a}$ & $>0.05$ & 1.53 \\
\hline Cys & n.d. & $0.07 \pm 0.04$ & $>0.05$ & - \\
\hline Ethan & $0.78 \pm 0.14 \mathrm{a}$ & $0.95 \pm 0.01 \mathrm{a}$ & $>0.05$ & 0.87 \\
\hline GABA & n.d. & $0.02 \pm 0.00$ & $>0.05$ & - \\
\hline Gln & $1.55 \pm 0.22 \mathrm{a}$ & $2.74 \pm 0.08 \mathrm{a}$ & $>0.05$ & 2.15 \\
\hline Glu & $3.36 \pm 0.01 \mathrm{a}$ & $4.88 \pm 0.04 \mathrm{a}$ & $>0.05$ & 4.12 \\
\hline Gly & $4.29 \pm 0.00 \mathrm{a}$ & $4.78 \pm 0.03 \mathrm{a}$ & $>0.05$ & 4.54 \\
\hline Homocys & n.d. & n.d. & - & - \\
\hline 1-Mhis & $0.34 \pm 0.00 \mathrm{a}$ & $0.38 \pm 0.04 \mathrm{a}$ & $>0.05$ & 0.36 \\
\hline 3-Mhis & $0.18 \pm 0.01 \mathrm{a}$ & $0.20 \pm 0.02 \mathrm{a}$ & $>0.05$ & 0.19 \\
\hline Orn & $0.14 \pm 0.00 \mathrm{a}$ & $0.28 \pm 0.01 \mathrm{a}$ & $>0.05$ & 0.21 \\
\hline Pea & $4.15 \pm 0.07 \mathrm{a}$ & $2.99 \pm 0.10 \mathrm{a}$ & $>0.05$ & 3.57 \\
\hline Phser & $1.48 \pm 0.04 \mathrm{a}$ & $2.04 \pm 0.13 \mathrm{a}$ & $>0.05$ & 1.76 \\
\hline Pro & $0.16 \pm 0.01 \mathrm{a}$ & $0.44 \pm 0.02 \mathrm{a}$ & $>0.05$ & 0.30 \\
\hline Sarc & $0.11 \pm 0.01 \mathrm{a}$ & $0.17 \pm 0.03 \mathrm{a}$ & $>0.05$ & 0.14 \\
\hline Ser & $1.59 \pm 0.00 \mathrm{a}$ & $1.66 \pm 0.06 \mathrm{a}$ & $>0.05$ & 1.63 \\
\hline Taur & $16.53 \pm 0.00 \mathrm{a}$ & $13.30 \pm 0.05 a$ & $<0.05$ & 14.92 \\
\hline Tyr & $0.47 \pm 0.00 \mathrm{a}$ & $0.64 \pm 0.10 \mathrm{a}$ & $>0.05$ & 0.56 \\
\hline Total & 44.8 & 47.59 & - & 46.15 \\
\hline Urea & $53.44 \pm 5.04$ & $82.32 \pm 8.32$ & $<0.001$ & 67.88 \\
\hline
\end{tabular}

\# Free and protein amino acids. n.d., not detected. Three-letter code has been used. Values followed by different letters within a row are significantly $(p<0.05)$ different by Bonferroni post-test. Protein amino acids are highlighted in bold.

\subsection{Amino Acid Content of Cow, Sheep and Goat Raw Milk from 'Alto Casertano'}

Raw milk got from some mammalian species is one of the most important sources of proteins for human nutrition [23]. The total amino acid content (free plus protein) per $100 \mathrm{~g}$ of proteins from cow, sheep and goat milk is reported in Table 7. In particular, comparing the total amino acid content among the three species raised in the Ailano and 
Valle Agricola (Alto Casertano) territories, no qualitative differences were observed among cow, sheep and goat 'Alto Casertano' raw milk, while quantitative differences were found. Glx (glutamic acid + glutamine) was by far the most abundant among the total amino acids (about $21.67 \%, 19.69 \%$ and $19.71 \%$ for cow, sheep and goat raw milk, respectively). Leucine, lysine and Asx (aspartic acid + asparagine) content was quite abundant (about 7-8\%) in the three different species, while the proline content is the most abundant in sheep raw milk $(9.31 \%)$ with respect to goat and cow raw milk ( $8.94 \%$ and $6.5 \%$, respectively). Furthermore, the content of other amino acids did not exceed $5 \%$ of the total protein content; in particular, tyrosine, serine, alanine, arginine, isoleucine and histidine content were higher in cow and sheep raw milk than in goat raw milk.

Table 7. Total amino acid composition of 'Alto Casertano' raw milk samples. Values are means from 'Ailano' and 'Valle Agricola' milk and are expressed as g per $100 \mathrm{~g}$ of total protein.

\begin{tabular}{|c|c|c|c|}
\hline \multicolumn{4}{|c|}{ Total Amino Acid Composition of Alto Casertano Milk } \\
\hline Amino Acid & Cow & Sheep & Goat \\
\hline \multicolumn{4}{|c|}{ Essential amino acids } \\
\hline His & 3.66 & 2.52 & 2.34 \\
\hline Ile & 4.01 & 4.22 & 3.93 \\
\hline Leu & 8.81 & 7.91 & 8.23 \\
\hline Lys & 7.79 & 7.47 & 7.15 \\
\hline Met & 2.91 & 2.80 & 2.65 \\
\hline Phe & 4.67 & 4.76 & 4.75 \\
\hline Thr & 4.87 & 5.06 & 5.47 \\
\hline $\operatorname{Trp}$ & n.d. & n.d. & n.d. \\
\hline Val & 4.79 & 5.01 & 5.22 \\
\hline \multicolumn{4}{|c|}{ Non-essential amino acids } \\
\hline Ala & 3.31 & 3.64 & 2.92 \\
\hline Arg & 3.33 & 3.40 & 2.75 \\
\hline Asx & 7.49 & 7.27 & 6.55 \\
\hline Cys & 0.87 & 1.06 & 0.87 \\
\hline Glx & 21.67 & 19.69 & 19.71 \\
\hline Gly & 1.80 & 1.74 & 1.52 \\
\hline Pro & 6.58 & 9.31 & 8.94 \\
\hline Ser & 5.96 & 5.69 & 5.16 \\
\hline Tyr & 5.00 & 5.04 & 3.78 \\
\hline
\end{tabular}

For protein amino acids, a three-letter code has been used. n.d., not determined.

In addition, the content of valine, threonine and phenylalanine was higher in sheep and goat raw milk than in cow raw milk, while glycine content was the same for the three species. Moreover, the amount of methionine and cysteine in the three species did not exceed the $4 \%$ of total protein, confirming the low level of sulfur amino acids. These data show that the quantity of essential amino acids in the three different types of raw milk is about $40 \%$ of the total proteins, confirming the good protein quality of this food.

The results discussed in this work demonstrate how the amino acid content of the milks analyzed changes among the different species, which is also shown by the radar chart of the average milk amino acid composition from three different mammal species (cow, sheep and goat), raised in two localities of 'Alto Casertano' (Campania region, Italy) (Figure 3).

Furthermore, in Figure 4, the total amino acid profiles obtained from raw milks were compared with the milk values reported by Claeys et al., showing that the amino acid profiles of cow, sheep and goat raw milk are similar to those previously reported [22]. On the other hand, the amino acid content of mountain milk (expressed as $\mathrm{g} / 100 \mathrm{~g}$ of milk) is higher than that of the milk values reported by Claeys et al. [22]. 


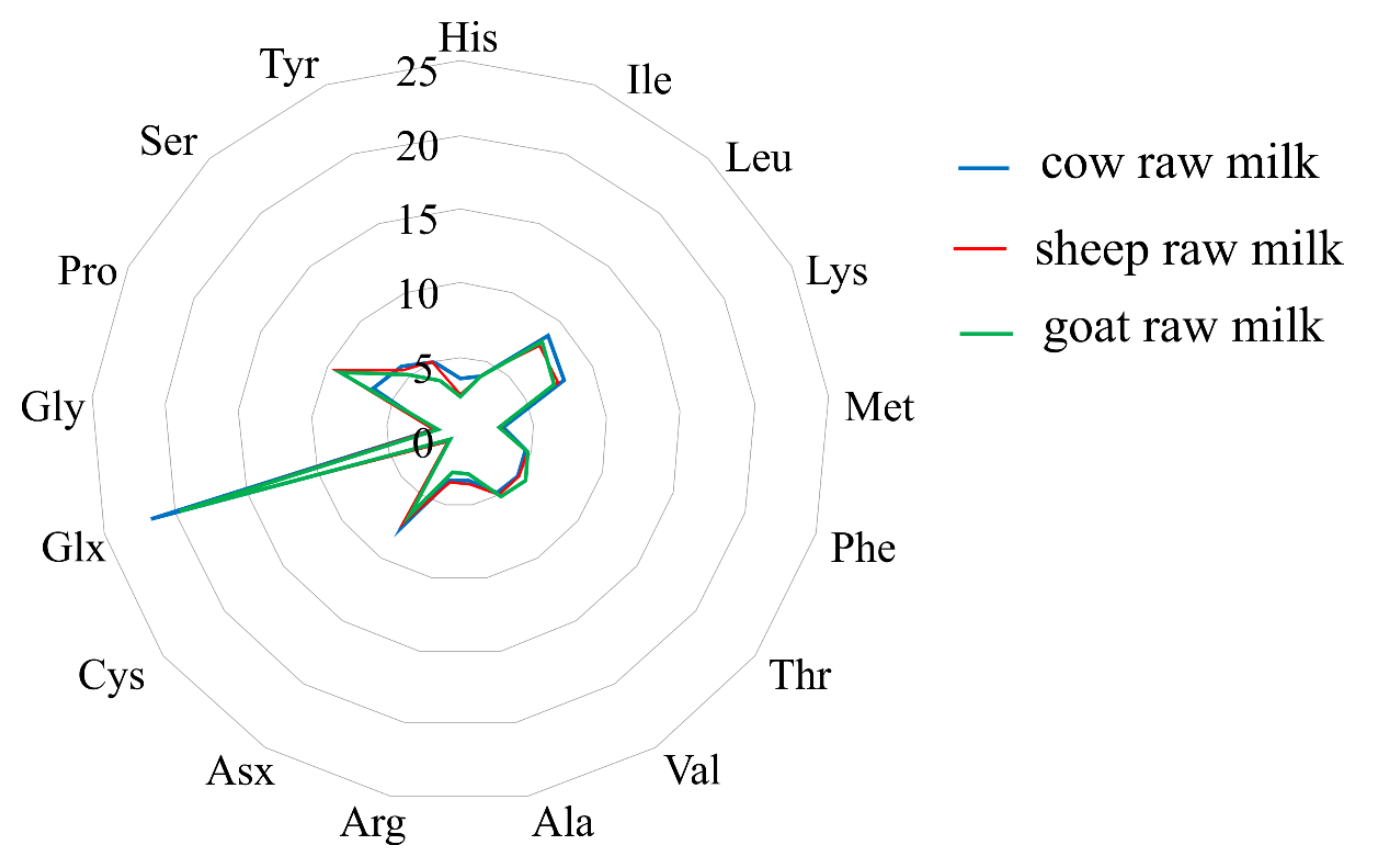

Figure 3. Radar graph of the average raw milk amino acid composition from three different mammal species (cow, sheep and goat), raised in two localities of 'Alto Casertano'.
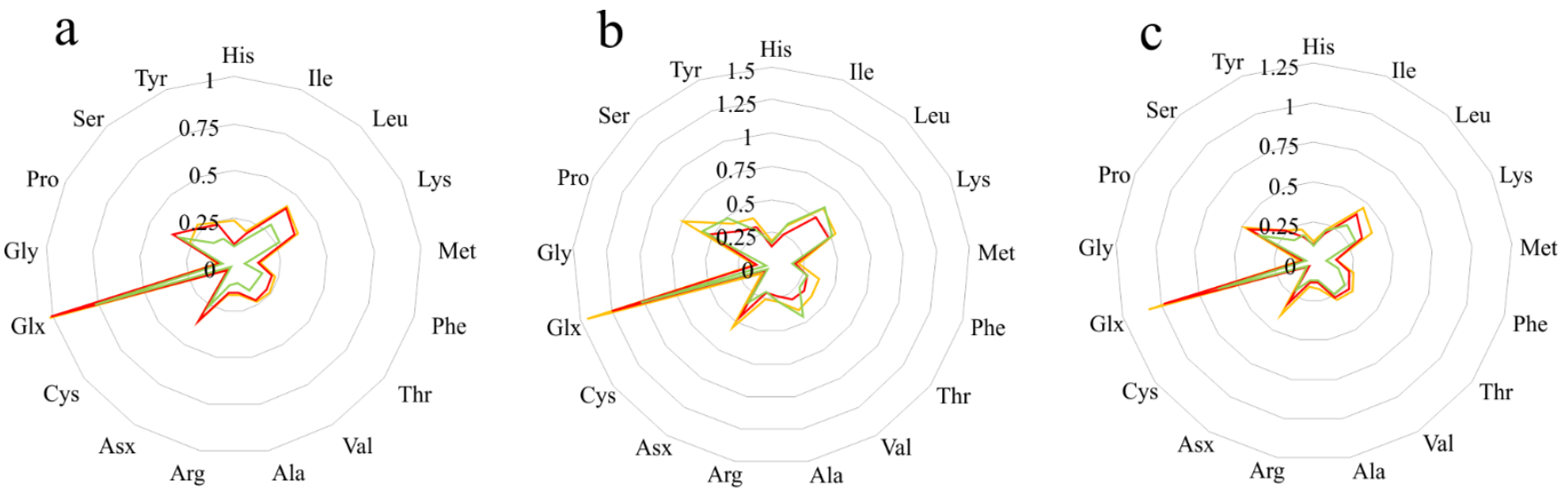

\section{- Ailano - Valle Agricola - Reference}

Figure 4. Radar graphs of the total amino acid content from the three types of milk analyzed, sampled in Ailano and Valle Agricola mountain localities, in comparison with the reference values [22]. (a) cow raw milk, (b) sheep raw milk and (c) goat raw milk.

On the other hand, comparing the free amino acid content among cow, sheep and goat raw milk, raised in the 'Alto Casertano', qualitative and quantitative differences were found. The total free amino acid content per $100 \mathrm{~g}$ of cow, sheep and goat 'Alto Casertano' raw milk was 21.33, 21.86 and $46.15 \mathrm{mg}$, respectively (Table 8 ).

Glutamic acid is the most abundant among the free protein amino acids in cow raw milk ( $\sim 43 \%$ of total), while it represents $\sim 14 \%$ and $\sim 9 \%$ in sheep and goat raw milk, respectively. Vice versa, the most abundant free protein amino acids in sheep and goat raw milk were tyrosine and glycine, respectively. In particular, tyrosine represents about $22 \%$ of total free amino acids in sheep raw milk and about $1 \%$ in both cow and goat raw milk. Glycine represents about $10 \%$ of total free amino acids in goat raw milk and about 7 and $5 \%$ of total free amino acids in cow and sheep raw milk, respectively. Furthermore, glutamine is present only in goat raw milk ( $5 \%$ of total free amino acids), while asparagine and alanine represent about $6 \%$ and $3 \%$ of total free amino acids in goat raw milk and about $4 \%$ 
and $2 \%$ in cow and sheep raw milk, respectively. Moreover, the amount of free essential amino acids, was about $7 \%$ of total free amino acids in both cow and goat raw milk and $12 \%$ of total free amino acids in sheep raw milk vs. $40 \%$ of total amino acids (free plus protein), not significant as a contribution in a human diet.

Table 8. Free amino acid composition of 'Alto Casertano' milk samples. Values are means from 'Ailano' and 'Valle Agricola' milk and are expressed as mg per $100 \mathrm{~g}$ of milk.

\begin{tabular}{|c|c|c|c|}
\hline \multicolumn{4}{|c|}{ Free Amino Acid Composition of 'Alto Casertano' Milk } \\
\hline Amino Acid ${ }^{a}$ & Cow & Sheep & Goat \\
\hline \multicolumn{4}{|c|}{ Essential amino acids } \\
\hline His & 0.06 & 0.11 & 0.11 \\
\hline Ile & 0.20 & 0.22 & 0.28 \\
\hline Leu & 0.19 & 0.43 & 0.46 \\
\hline Lys & 0.41 & 0.27 & 0.65 \\
\hline Met & - & - & - \\
\hline Phe & 0.24 & 0.73 & 0.44 \\
\hline Thr & 0.17 & 0.46 & 0.69 \\
\hline $\operatorname{Trp}$ & - & 0.00 & - \\
\hline Val & 0.21 & 0.42 & 0.81 \\
\hline \multicolumn{4}{|c|}{ Non-essential amino acids } \\
\hline AAAA & 0.13 & 0.12 & 0.11 \\
\hline Aaba & 0.13 & - & 0.21 \\
\hline Ala & 0.91 & 0.60 & 1.26 \\
\hline Arg & 0.34 & 0.71 & 0.86 \\
\hline Asn & 0.28 & 0.77 & 2.75 \\
\hline Asp & 0.70 & 0.33 & 0.60 \\
\hline$\beta$-Ala & 0.13 & 0.19 & 0.13 \\
\hline Car & - & 0.09 & - \\
\hline Citr & 0.14 & 0.47 & 1.53 \\
\hline Cys & - & - & - \\
\hline Ethan & 1.16 & 1.83 & 0.87 \\
\hline GABA & - & 0.01 & - \\
\hline Gln & - & - & 2.15 \\
\hline Glu & 9.07 & 2.98 & 4.12 \\
\hline Gly & 1.52 & 1.12 & 4.54 \\
\hline Homocys & - & - & - \\
\hline 1-Mhis & - & 0.31 & 0.36 \\
\hline 3-Mhis & - & 0.14 & 0.19 \\
\hline Orn & 0.08 & 0.15 & 0.21 \\
\hline Pea & 1.57 & 0.29 & 3.57 \\
\hline Phser & 1.35 & 1.63 & 1.76 \\
\hline Pro & 0.48 & 0.28 & 0.30 \\
\hline Sarc & 0.14 & 0.15 & 0.14 \\
\hline Ser & 0.30 & 0.28 & 1.63 \\
\hline Taur & 1.38 & 2.10 & 14.92 \\
\hline Tyr & 0.11 & 4.72 & 0.56 \\
\hline Total & 21.33 & 21.86 & 46.15 \\
\hline
\end{tabular}

a, free and protein amino acids. Three-letter code has been used. Protein amino acids are highlighted in bold.

The analysis also revealed the presence of quali-quantitative differences in non-protein amino acids. In particular, the amount of non-protein amino acids in cow, sheep and goat raw milk was $29 \%, 34 \%$ and $52 \%$ of total free amino acids, respectively. In particular, $\mathrm{L}-\alpha$-aminobutyric acid is present only in cow and goat raw milk ( $2 \%$ and $1 \%$ of total nonprotein amino acids, respectively), while 1-Methylhistidine and 3-Methylhistidine were present only in sheep and goat raw milk ( 1.0\% and $0.6 \% 1$-Mhis; $\sim 0.6 \%$ and $0.4 \% 3$-Mhis of total non-protein amino acids, respectively). Finally, L-carnitine is present only sheep raw milk ( $\sim .4 \%$ of total non-protein amino acids). Furthermore, taurine is an aminosulfonic 
acid, derived from methionine and cysteine and in strict sense, it is not an amino acid. Taurine is an essential nutrient for the infant due to its insufficient endogenous synthesis. Taurine may act as a membrane stabilizer and growth modulator and plays a role in the formation of bile acids, which facilitates lipid digestion and absorption [24].

The data obtained in this study highlight that goat raw milk is a good source of the amino acid taurine, which represents about $32 \%$ of total free amino acids, as previously reported [25]; meanwhile, the content of taurine in cow and sheep raw milk was about $7 \%$ and $10 \%$, respectively.

Finally, as shown in the radar graphs (Figure 5), a comparison of the average free amino acid profile from cow, sheep and goat raw milk analyzed reveals that the free amino acid footprint of the three milk types changes among the species analyzed.

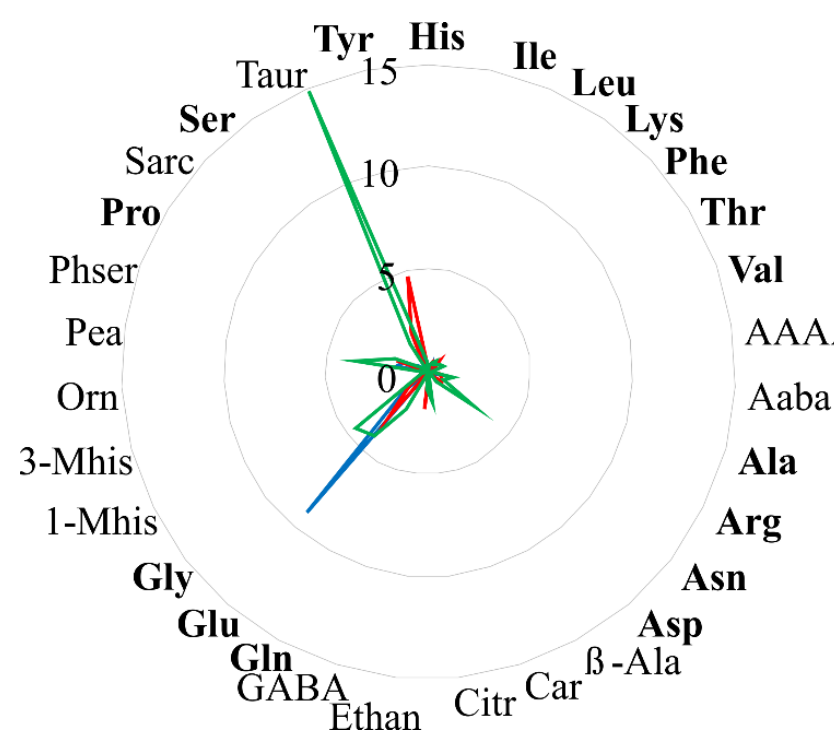

Figure 5. Radar graph of the average free amino acid profile from cow, sheep and goat raw milk analyzed samples.

\section{Conclusions}

Consumers associate the value and quality of raw milk with uncontaminated breeding places, such as mountain territories, where the animals are kept in a semi-wild regime. In this scenario, confirming the goodness of raw milk from mountainous areas can be useful to encourage local production and, subsequently, commercialization. Raw milk is a reservoir of high-quality proteins and the best source of nutrition for nearly all infants, containing all nutrients necessary for the growth and development of newborn [26]. Moreover, raw milk from different species (e.g., cow, goat and sheep) continues to be part of human nutrition in adult life. For these reasons, the free and total amino acid profile of milk from different species plays a key role for both milk producers and processors, as well as for consumers, in order to reach innovative product design, versatility, taste and functionality.

In light of this, we investigated the total protein content (caseins plus whey proteins) and total and free amino acid profiles of raw milk from cow, sheep and goat raised in Ailano and Valle Agricola territories, two mountain localities of 'Alto Casertano' (Campania region, Italy). In particular, the three raw milk samples analyzed showed higher total amino acid content with respect to milk values reported by Claeys et al. [22].

On the other hand, free amino acid profiles from cow, sheep and goat raw milks are characteristic and can be used as a hallmark of these species.

Overall, the higher quality of the three different mountain raw milk analyzed samples from Ailano and Valle Agricola could justify the possibility of the highest retail price of this product and its derivatives, encouraging the local farmers to increase milk production in order to provide adequate incomes for the local small farms, converting their economy from subsistence incomes to profit ones. 
Author Contributions: N.L. and S.R. performed protein quantization and amino acid analyses. A.D.M. conceived the study and wrote the paper. All authors have read and agreed to the published version of the manuscript.

Funding: This work was supported by funds from PSR Campania 2014-2020 (Misura 16-Tipologia di Intervento 16.1.1-Azione 2-Progetto 2-“NATURA LATTEA".

Institutional Review Board Statement: Not applicable.

Informed Consent Statement: Not applicable.

Data Availability Statement: Raw data can be provided by the corresponding author on request.

Acknowledgments: This study was made possible by care and abnegation of all participants, despite the absence of dedicated funds and chronic difficulties afflicting the Italian scientific community.

Conflicts of Interest: The authors have no conflict of interest.

\section{Abbreviations}

For the amino acids, the standard tree-letter code has been used.

\section{References}

1. O'Mahony, J.A.; Fox, P.F. Milk: An Overview. In Milk Proteins from Expression to Food; Singh, H., Boland, M., Thompson, A., Eds.; Elsevier: London, UK, 2014. [CrossRef]

2. Pouliot, Y.; Gauthier, S. Milk growth factors as health products: Some technological aspects. Int. Dairy J. 2006, 16, 1415-1420. [CrossRef]

3. Guetouache, M.; Guessas, B.; Medjekal, S. Composition and Nutritional Value of Raw Milk. Issues Biol. Sci. Pharm. Res. 2014, 2, 115-122.

4. Bhat, Z.F.; Bhat, H. Milk and Dairy Products as Functional Foods: A Review. Int. J. Dairy Sci. 2011, 6, 1-12. [CrossRef]

5. Fox, P.F.; Uniacke-Lowe, T.; McSweeney, P.L.H.; O'Mahony, J.A. Milk Proteins. In Dairy Chemistry and Biochemistry; Springer International Publishing: Cham, Switzerland, 2015; pp. 145-239. [CrossRef]

6. Barłowska, J.; Szwajkowska, M.; Litwińczuk, Z.; Król, J. Nutritional Value and Technological Suitability of Milk from Various Animal Species Used for Dairy Production. Compr. Rev. Food Sci. Food Saf. 2011, 10, 291-302. [CrossRef]

7. Addeo, F.; Debry, G.; Paquot, M.; Sachet, P.; Schlimme, E.; Soustre, Y. Le Proteine del Latte e Derivati; MASSON: Milano, Italy, 1994.

8. Bramanti, E.; Sortino, C.; Onor, M.; Beni, F.; Raspi, G. Separation and determination of denatured alpha(s1)-, alpha(s2)-, betaand kappa-caseins by hydrophobic interaction chromatography in cows', ewes' and goats' milk, milk mixtures and cheeses. J. Chromatogr. A 2003, 994, 59-74. [CrossRef]

9. Balthazar, C.F.; Pimentel, T.C.; Ferrão, L.L.; Almada, C.N.; Santillo, A.; Albenzio, M.; Mollakhalili, N.; Mortazavian, A.M.; Nascimento, J.S.; Silva, M.C.; et al. Sheep Milk: Physicochemical Characteristics and Relevance for Functional Food Development. Compr. Rev. Food Sci. Food Saf. 2017, 16, 247-262. [CrossRef] [PubMed]

10. Fox, P.F.; Guinee, T.P.; Cogan, T.M.; McSweeney, P.L.H. Chemistry of Milk Constituents. In Fundamentals of Cheese Science; Springer: Boston, MA, USA, 2017; pp. 71-104. [CrossRef]

11. Hambræus, L.; Lönnerdal, B. Nutritional Aspects of Milk Proteins. In Advanced Dairy Chemistry—1 Proteins: Part A/Part B; Fox, P.F., McSweeney, P.L.H., Eds.; Springer: Boston, MA, USA, 2003; pp. 605-645. [CrossRef]

12. Caroli, A.M.; Chessa, S.; Erhardt, G.J. Invited review: Milk protein polymorphisms in cattle: Effect on animal breeding and human nutrition. J. Dairy Sci. 2009, 92, 5335-5352. [CrossRef] [PubMed]

13. Heck, J.M.; Schennink, A.; van Valenberg, H.J.; Bovenhuis, H.; Visker, M.H.; van Arendonk, J.A.; van Hooijdonk, A.C. Effects of milk protein variants on the protein composition of bovine milk. J. Dairy Sci. 2009, 92, 1192-1202. [CrossRef] [PubMed]

14. Council, N.R. Protein and Amino Acids. In Recommended Dietary Allowances, 10th ed.; The National Academies Press: Washington, DC, USA, 1989; pp. 52-77. [CrossRef]

15. AOAC_976.05. AOAC Official Method 976.05. In Officinal Methods of Analysis of AOAC International, 17th ed.; Horwitz, W., Ed.; AOAC International: Gaithersburg, MD, USA, 2000; Volume 1, p. 23.

16. Landi, N.; Pacifico, S.; Ragucci, S.; Di Giuseppe, A.M.; Iannuzzi, F.; Zarrelli, A.; Piccolella, S.; Di Maro, A. Pioppino mushroom in southern Italy: An undervalued source of nutrients and bioactive compounds. J. Sci. Food Agric. 2017, 97, 5388-5397. [CrossRef] [PubMed]

17. Di Maro, A.; Dosi, R.; Ferrara, L.; Rocco, M.; Sepe, J.; Ferrari, G.; Parente, A. Free amino acid profile in Malus domestica cv Annurca apples from the Campania region and other Italian vegetables. Aust. J. Crop Sci. 2011, 5, 154-161.

18. Ozols, J. Amino acid analysis. Methods Enzymol. 1990, 182, 587-601. [PubMed]

19. Moore, S.; Spackman, D.H.; Stein, W.H. Automatic recording apparatus for use in the chromatography of amino acids. Fed. Proc. 1958, 17, 1107-1115. [PubMed] 
20. Aitken, A.; Learmonth, M. Performic Acid Oxidation. In The Protein Protocols Handbook; Walker, J.M., Ed.; Humana Press: Totowa, NJ, USA, 2002; pp. 457-458. [CrossRef]

21. DePeters, E.J.; Ferguson, J.D. Non protein nitrogen and protein distribution in the milk of cows. J. Dairy Sci. 1992, 75, 3192-3209. [CrossRef]

22. Claeys, W.; Verraes, C.; Cardoen, S.; Block, J.; Huyghebaert, A.; Raes, K.; Dewettinck, K.; Herman, L. Consumption of raw or heated milk from different species: An evaluation of the nutritional and potential health benefits. Food Control 2014, 42, 188-201. [CrossRef]

23. Pereira, P.C. Milk nutritional composition and its role in human health. Nutrition 2014, 30, 619-627. [CrossRef]

24. Lawrence, R.A.; Lawrence, R.M. Chapter 4-Biochemistry of Human Milk. In Breastfeeding, 7th ed.; Lawrence, R.A., Lawrence, R.M., Eds.; W.B. Saunders: Philadelphia, PA, USA, 2011; pp. 98-152. [CrossRef]

25. Tripaldi, C.; Martillotti, F.; Terramoccia, S. Content of taurine and other free amino acids in milk of goats bred in Italy. Small Ruminant Res. 1998, 30, 127-136. [CrossRef]

26. Lonnerdal, B.; Bhatia, J.; Shamir, R.; Vandenplas, Y. Protein in Neonatal and Infant Nutrition: Recent Updates, 1st ed.; Kanger: Beijing, China, 2016; Volume 86. 